Communications in Physics, Vol. 24, No.3S1 (2014), pp. 45-50

DOI: $10.15625 / 0868-3166 / 24 / 3 S 1 / 5135$

\title{
HALL EFFECT IN THE DOPED SEMICONDUCTOR SUPERLATTICE WITH AN IN-PLANE MAGNETIC FIELD UNDER INFLUENCE OF AN INTENSE ELECTROMAGNETIC WAVE
}

\author{
NGUYEN QUANG BAU \\ Faculty of Physics, Hanoi University of Science, Vietnam National University, Hanoi, Vietnam \\ BUI DINH HOI \\ Department of Physics, National University of Civil Engineering, Hanoi, Vietnam
}

TRAN CONG PHONG

Center for Theoretical and Computational Physics, Hue University's College of Education,

Hue city, Vietnam

E-mail: hoibd@nuce.edu.vn

Received 04 April 2014

Accepted for publication 24 August 2014

\begin{abstract}
The Hall effect is studied theoretically in a doped semiconductor superlattice (DSSL) subjected to a crossed dc electric field and magnetic field in the presence of an intense electromagnetic wave (EMW). By using the quantum kinetic equation for electrons interacting with acoustic phonons at low temperature, we obtain expressions for the magnetoresistance as well as the Hall coefficient in dependence on the external fields and characteristic parameters of the DSSL. Analytical results are numerically evaluated for the GaAs:Si/GaAs:Be DSSL. The dependence of the magnetoresistance on the magnetic field is consistent with the result obtained for some two-dimensional electron systems. The Hall coefficient depends weakly on the magnetic field and its value in the presence of the EMW is smaller than that of the case without EMW.

Keywords: Hall effect, magnetoresistance, electron-phonon interaction, doping superlattice.
\end{abstract}

\section{INTRODUCTION}

A doped semiconductor superlattice (DSSL) is formed by layers of n-type and p-type semiconductors arranged alternatively between an intrinsic semiconductor (so called n-i-p-i superlattice). The most important advantage of DSSLs is the possibility of adjustment of their parameters to get long lifetimes, high carrier mobility. The electrical and optical properties of the DSSL structure may be modulated within a wide range of values. This kind of low-dimensional system, has attracted much attention, both theoretically and experimentally.

In two-dimensional (2D) electron systems, the Hall effect has been studied in many aspects (see [1] for recent reviews). However, most of previous works only considered the case when an electromagnetic wave (EMW) was absent and at the temperature that electron - electron and electron - impurity interactions to be dominant (conditions for the integer and fractional quantum Hall effect). The propagation of an EMW in materials leads to the change in the scattering (C)2014 Vietnam Academy of Science and Technology 
probability of carriers, and thus, leads to their unusual behaviors in comparison to the case of the absence of the EMW. In some recent works, we have used the quantum kinetic equation method to study the influence of a high-frequency EMW on the Hall effect in parabolic quantum wells [2], doping semiconductor superlattices [3] at high temperatures, and in rectangular quantum wells at low temperature [4]. We also have used this method to investigate the acoustoelectric and magnetoacoustoelectric effects $[5,6]$. The Hall effect in low-dimensional systems, especially in 2D semiconductor systems, under the influence of a high-frequency EMW still remains problems to study, especially by analytical and computational methods.

In this work, by using the quantum kinetic equation method, we study the Hall effect in the DSSL subjected to an in-plane magnetic field in the presence of an intense EMW. We only consider the case of low temperatures when the electron - acoustic phonon interaction is assumed to be dominant and electron gas is weakly degenerate. We derive analytical expressions for the magnetoresistance (MR) and the Hall coefficient (HC) taking account of arbitrary transitions between the energy levels. The analytical result is numerically evaluated and graphed for the GaAs:Si/GaAs:Be DSSL. In the next section, we write out the electronic structure and the Hamiltonian of the system. Analytical results of the calculation are presented in Sec. III. Numerical results, discussion, and remarks are given in Sec. IV.

\section{HAMILTONIAN OF ELECTRON - PHONON SYSTEM}

We consider a simple model of the DSSL in which electron gas is confined by the superlattice potential along the $z$-direction and free in the $(x-y)$ plane. The motion of an electron is limited in each layer of the system and its energy spectrum is quantized into discrete levels in the $z$-direction. According to K. Ploog and G. H. Dohler [7], in an ideal model, the DSSL can be treated as a multiquantum well structure in which the confinement potential in each well is parabolic. In most cases of interest it is justified to neglect the interaction between neighboring potential wells. Therefore, the single-particle wave function and corresponding eigen-energy in the DSSL has the same mathematical forms with those obtained for a harmonic oscillator. If the DSSL is subjected to a crossed dc electric field $\vec{E}_{1}=\left(0,0, E_{1}\right)$ and magnetic field $\vec{B}=(0, B, 0)$, the single-particle wave function and corresponding eigenenergy of an electron in a single potential well are given by [8]

$$
\begin{gathered}
\left|N, k_{x}\right\rangle=\psi_{0} e^{i \vec{k}_{\perp} \vec{r}_{\perp}} \Phi_{N}\left(z-z_{0}\right) \\
\varepsilon_{N}\left(\vec{k}_{x}\right)=\left(N+\frac{1}{2}\right) \hbar \tilde{\omega}+\frac{1}{2 m_{e}}\left[\hbar^{2} k_{x}^{2}-\left(\frac{\hbar k_{x} \omega_{c}+e E_{1}}{\tilde{\omega}}\right)^{2}\right], \quad N=0,1,2, \ldots,
\end{gathered}
$$

where $\psi_{0}$ is a normalization factor, $m_{e}$ and $e$ are the effective mass and the charge of a conduction electron, respectively, $\vec{k}_{\perp}=\left(k_{x}, k_{y}\right)$ is its wave vector in the $(x-y)$ plane, $\vec{r}_{\perp}=(x, y)$, $\vec{k}_{x}=\left(k_{x}, 0,0\right), z_{0}=\left(\hbar k_{x} \omega_{c}+e E_{1}\right) / m_{e} \tilde{\omega}^{2}, \tilde{\omega}^{2}=\omega_{c}^{2}+\omega_{p}^{2}, \omega_{c}$ is the cyclotron frequency and $\omega_{p}=\left(e^{2} n_{D} / \kappa_{0} m_{e}\right)^{1 / 2}$ is the plasma frequency characterizing for the DSSL confinement in the $z$-direction, where $\kappa_{0}$ is the electronic constant (vacuum permittivity) and $n_{D}$ is the doping concentration, and $[8,9]$

$$
\Phi_{N}\left(z-z_{0}\right)=\sqrt{\frac{1}{2^{N} N ! \sqrt{\pi} \ell_{z}}} \exp \left(-\frac{\left(z-z_{0}\right)^{2}}{2 \ell_{z}^{2}}\right) H_{N}\left(\frac{z-z_{0}}{\ell_{z}}\right)
$$


with $H_{N}(z)$ being the Hermite polynomials of $N^{\text {th }}$ order and $\ell_{z}=$ $\left(\hbar /\left(m_{e} \omega_{p}\right)\right)^{1 / 2}$. In the limit of $n_{D} \rightarrow$ 0 , the energy spectrum (2) becomes the one obtained in normal semiconductors by Kahn and Frederikse [10].

In the presence of an intense EMW with electric field vector $\vec{E}=$ $\left(E_{0} \sin (\Omega t), 0,0\right)\left(E_{0}\right.$ and $\Omega$ are the amplitude and the frequency of the EMW, respectively), the Hamiltonian of the electron-acoustic phonon system in the DSSL, in the second quantization representation, can be written as

$$
\begin{gathered}
H=H_{0}+U, \\
H_{0}=\sum_{N, \vec{k}_{x}} \varepsilon_{N}\left(\vec{k}_{x}-\frac{e}{\hbar c} \vec{A}(t)\right) a_{N, \vec{k}_{x}}^{+} a_{N, \vec{k}_{x}}+\sum_{\vec{q}} \hbar \omega_{\vec{q}} b_{\vec{q}}+b_{\vec{q}}, \\
U=\sum_{N, N^{\prime}} \sum_{\vec{q}, \vec{k}_{x}} D_{N, N^{\prime}}(\vec{q}) a_{N^{\prime}, \vec{k}_{x}+\vec{q}_{x}}^{+} a_{N, \vec{k}_{x}}\left(b_{\vec{q}}+b_{-\vec{q}}^{+}\right),
\end{gathered}
$$

where $\left|N, \vec{k}_{x}\right\rangle$ and $\left|N^{\prime}, \vec{k}_{x}+\vec{q}_{x}\right\rangle$ are electron states before and after scattering, $\hbar \omega_{\vec{q}}$ is the energy of a phonon with the wave vector $\vec{q}=\left(q_{x}, q_{y}, q_{z}\right), a_{N, \vec{k}_{x}}^{+}$and $a_{N, \vec{k}_{x}}\left(b_{\vec{q}}^{+}\right.$and $\left.b_{\vec{q}}\right)$ are the creation and annihilation operators of electron (phonon), respectively, $\vec{A}(t)$ is the vector potential of the EMW, and $D_{N, N^{\prime}}(\vec{q})$ is the matrix element of interaction, given by $[9,11]$

$$
\left|D_{N, N^{\prime}}(\vec{q})\right|^{2}=\left|C_{\vec{q}}\right|^{2}\left|I_{N, N^{\prime}}\left( \pm q_{z}\right)\right|^{2}
$$

where $C_{\vec{q}}$ is the electron-phonon interaction constant which depends on the interacting mechanism, $I_{N, N^{\prime}}\left( \pm q_{z}\right)$ is the form factor of electron, given by

$$
I_{N, N^{\prime}}\left( \pm q_{z}\right)=\sum_{\wp=1}^{s_{0}} \int_{0}^{d} e^{ \pm i q_{z} d} \varphi_{N}(z-\wp d) \varphi_{N^{\prime}}(z-\wp d) d z,
$$

with $d$ is the period and $s_{0}$ is the number of periods of the DSSL. In Fig. 1, we show the real and the imagine parts of the form factor versus the component $q_{z}$ in two first mini Brillouin zones.

\section{ANALYTICAL RESULTS}

By using Hamiltonian (1) and procedures as in the previous works [2-6], we obtain an equation for the partial current density in the single (constant) relaxation time approximation. To do this, we assume that the electrons system is degenerate and the distribution function has the form of Heaviside step function. For the electron - acoustic phonon interaction, $\hbar \omega_{\vec{q}}=\hbar v_{s} q$ and [11]

$$
\left|C_{\vec{q}}\right|^{2}=\frac{\xi^{2} q}{2 \rho v_{s} V_{0}}
$$


where $v_{s}, \xi, \rho$, and $V_{0}$ are, respectively, the sound velocity, the acoustic deformation potential, the mass density, and the normalization volume of the specimen. After some manipulation, we obtain the expression for the conductivity tensor:

$$
\sigma_{i m}=\frac{e \tau}{m_{e}} \frac{b \tau}{1+\omega_{c}^{2} \tau^{2}}\left(\delta_{i j}-\omega_{c} \tau \varepsilon_{i j k} h_{k}+\omega_{c}^{2} \tau^{2} h_{i} h_{j}\right) \delta_{j l}\left[\delta_{\ell m}-\omega_{c} \tau \varepsilon_{\ell m p} h_{p}+\omega_{c}^{2} \tau^{2} h_{\ell} h_{m}\right]
$$

where $\tau$ is the momentum relaxation time, $\delta_{i j}$ is the Kronecker delta, $\varepsilon_{i j k}$ being the antisymmetrical Levi - Civita tensor, the Latin symbols $i, j, k, m, l, p$ stand for the components $x, y, z$ of the Cartesian coordinates,

$$
\begin{aligned}
& b=\frac{2 e L_{x} A}{\sqrt{2} \pi^{2} m_{e} \hbar} \sum_{N, N^{\prime}} I\left(N, N^{\prime}\right)\left[b_{1}+b_{2}+b_{3}+b_{4}\right], \\
& b_{1}=\frac{1}{\sqrt{\Delta_{k} \Delta_{q}^{(1)}}}\left\{k_{0}^{+}\left\{\left(q_{1(+)}^{+}\right)^{3}\left[\exp \left(\sqrt{2} \beta \hbar v_{s} q_{1(+)}^{+}\right)-1\right]^{-1}+\left(q_{1(+)}^{-}\right)^{3}\left[\exp \left(\sqrt{2} \beta \hbar v_{s} q_{1(+)}^{-}\right)-1\right]^{-1}\right\}\right. \\
& \left.+k_{0}^{-}\left\{\left(q_{1(-)}^{+}\right)^{3}\left[\exp \left(\sqrt{2} \beta \hbar v_{s} q_{1(-)}^{+}\right)-1\right]^{-1}+\left(q_{1(-)}^{-}\right)^{3}\left[\exp \left(\sqrt{2} \beta \hbar v_{s} q_{1(-)}^{-}\right)-1\right]^{-1}\right\}\right\}, \\
& b_{2}=-\frac{\theta}{2 \sqrt{\Delta_{k} \Delta_{q}^{(1)}}}\left\{k_{0}^{+}\left\{\left(q_{1(+)}^{+}\right)^{5}\left[\exp \left(\sqrt{2} \beta \hbar v_{s} q_{1(+)}^{+}\right)-1\right]^{-1}+\left(q_{1(+)}^{-}\right)^{5}\left[\exp \left(\sqrt{2} \beta \hbar v_{s} q_{1(+)}^{-}\right)-1\right]^{-1}\right\}\right. \\
& \left.+k_{0}^{-}\left\{\left(q_{1(-)}^{+}\right)^{5}\left[\exp \left(\sqrt{2} \beta \hbar v_{s} q_{1(-)}^{+}\right)-1\right]^{-1}+\left(q_{1(-)}^{-}\right)^{5}\left[\exp \left(\sqrt{2} \beta \hbar v_{s} q_{1(-)}^{-}\right)-1\right]^{-1}\right\}\right\}, \\
& b_{3}=\frac{\theta}{4 \sqrt{\Delta_{k} \Delta_{q}^{(2)}}}\left\{k_{0}^{+}\left\{\left(q_{2(+)}^{+}\right)^{5}\left[\exp \left(\sqrt{2} \beta \hbar v_{s} q_{2(+)}^{+}\right)-1\right]^{-1}+\left(q_{2(+)}^{-}\right)^{5}\left[\exp \left(\sqrt{2} \beta \hbar v_{s} q_{2(+)}^{-}\right)-1\right]^{-1}\right\}\right. \\
& \left.+k_{0}^{-}\left\{\left(q_{2(-)}^{+}\right)^{5}\left[\exp \left(\sqrt{2} \beta \hbar v_{s} q_{2(-)}^{+}\right)-1\right]^{-1}+\left(q_{2(-)}^{-}\right)^{5}\left[\exp \left(\sqrt{2} \beta \hbar v_{s} q_{2(-)}^{-}\right)-1\right]^{-1}\right\}\right\}, \\
& b_{4}=\frac{\theta}{4 \sqrt{\Delta_{k} \Delta_{q}^{(3)}}}\left\{k_{0}^{+}\left\{\left(q_{3(+)}^{+}\right)^{5}\left[\exp \left(\sqrt{2} \beta \hbar v_{s} q_{3(+)}^{+}\right)-1\right]^{-1}+\left(q_{3(+)}^{-}\right)^{5}\left[\exp \left(\sqrt{2} \beta \hbar v_{s} q_{3(+)}^{-}\right)-1\right]^{-1}\right\}\right. \\
& \left.+k_{0}^{-}\left\{\left(q_{3(-)}^{+}\right)^{5}\left[\exp \left(\sqrt{2} \beta \hbar v_{s} q_{3(-)}^{+}\right)-1\right]^{-1}+\left(q_{3(-)}^{-}\right)^{5}\left[\exp \left(\sqrt{2} \beta \hbar v_{s} q_{3(-)}^{-}\right)-1\right]^{-1}\right\}\right\}, \\
& I\left(N, N^{\prime}\right)=\int_{-\pi / d}^{+\pi / d}\left|I_{N, N^{\prime}}\left( \pm q_{z}\right)\right|^{2} d q_{z} .
\end{aligned}
$$

The MR and the $\mathrm{HC}$ are, respectively, given by

$$
\rho_{z z}(B)=\frac{\sigma_{z z}}{\sigma_{z z}^{2}+\sigma_{x z}^{2}} ; \quad R_{H}=-\frac{1}{B} \frac{\sigma_{x z}}{\sigma_{z z}^{2}+\sigma_{x z}^{2}}
$$




\section{NUMERICAL RESULTS}

In the following, we will give a deeper insight into analytical results by carrying out a numerical evaluation. For the computation, we consider the n-i-p-i DSSL of GaAs:Si/GaAs:Be with the following parameters [5-8]: $\xi=13.5 \mathrm{eV}, \rho=5320 \mathrm{~kg} \cdot \mathrm{m}^{-3}, v_{s}=5378 \mathrm{~m} \cdot \mathrm{s}^{-1}, \varepsilon_{F}=50$ $\mathrm{meV}, m_{e}=0.067 \times m_{0}\left(m_{0}\right.$ is the mass of a free electron $), d=100 \mathrm{~nm}, s_{0}=50$. For simplicity, we consider only the ground and the first-excited states: $N=0, N^{\prime}=1$.

Unfortunately, we are not aware of theoretical and experimental works on this problem other than [12-14] in which the MR and the HC are reported in bulk semiconductors [12], parabolic quantum wells [13], and compositional superlattices [14]. Therefore, we cannot compare our calculation with the experiment. However, we can compare our results with those obtained in [12-14] in some limits such as low and high doping concentration, weak and strong magnetic field.

Fig. 2 shows the relative change of the MR $\Delta \rho_{z z} / \rho_{z z}(0)=\rho\left(\rho_{z z}(B)-\rho_{z z}(0)\right) / \rho_{z z}(0)$ with magnetic field at $T=4.2 \mathrm{~K}$ and low doping concentration $\left(n_{D}=10^{20} \mathrm{~m}^{-3}\right)$. It is seen that there occurs the negative MR. In the range of weak magnetic field, the resistance monotonically decreases with increasing the magnetic field. This behavior is similar to the one classically observed in [12] for bulk semiconductors in which the parameter $\gamma=k_{B} T \tau / \hbar$ is small and in the InSb parabolic quantum well with weakly degenerate electron gas [13]. The relative change is largest at $B_{m} \approx 1.35 \mathrm{~T}$ and is about $45 \%$. In the range $B>B_{m}$ there is a monotonically increasing function of the magnetic field as observed in InSb quantum well. However, the most noticeable difference in this calculation is that the relative MR at low doping concentration is always negative at any magnetic field, i.e., the MR can not change its sign as the magnetic field increases. This can be explained as follow. In the calculation for parabolic quantum well [13], the authors have assumed a model of an infinite potential as we have done in this calculation. However, at low doping concentration, this approximation is maybe invalid, i.e, the confinement potential is not high enough for this assumption. From these results, we can conclude that at low doping concentration, the DSSL structure behaves as both bulk and 2D semiconductors.

In the range of high concentration, the assumption of infinite confinement potential with parabolic law is well done. In order to illustrate for this conclusion, in Fig. 3 we plot the MR (in arbitrary units) versus the magnetic field at the doping concentration of $7 \times 10^{24} \mathrm{~m}^{-3}$.

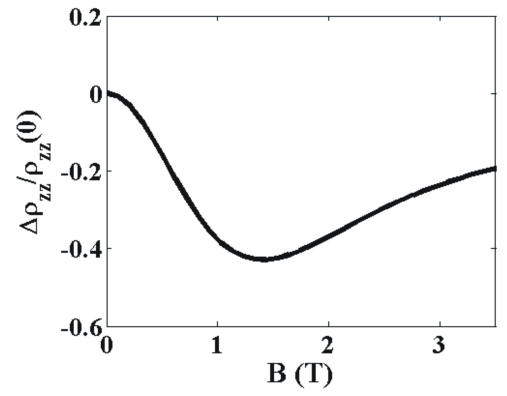

Fig. 2. The relative MR change as function of the magnetic field in the case of the absence of the EMW. Here, $n_{D}=10^{20} \mathrm{~m}^{-3}$, $\tau=10^{-12} s$, and $T=4.2 K$.

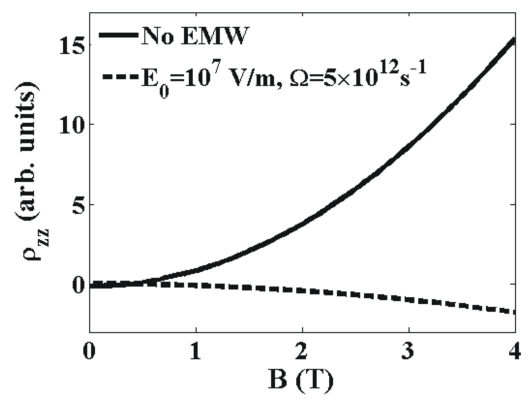

Fig. 3. The MR as function of the magnetic field at high doping concentration for two cases: the absence and the presence of the EMW. Here, $n_{D}=7 \times 10^{24} \mathrm{~m}^{-3}$, the other parameters are the same as in Fig. 1. 
The figure shows that there exists a value of the magnetic field at that the MR changes sign. As the magnetic field increases, the MR strongly increases. This behavior has been well observed in the InSb parabolic quantum well [13] and in GaAs/AlGaAs compositional semiconductor superlattices [14] at high magnetic field. It is also seen from Fig. 3 that the presence of the EMW changes the MR considerably. The MR is positive in the absence of the EMW, whereas it is negative if the EMW is swiched on.

Fig. 4 shows the dependence of the HC on the magnetic field. The $\mathrm{HC}$ is seen to weakly decrease with increasing the field. In the range of weak field, the HC is nearly independent of the magnetic field. This dependence is consistent with the result obtained for a degenerate electrons system [12] where $\gamma$ is small $(\gamma=5)$. It is usually observed in experiments. It is also seen that the presence of the intense EMW does not change the behavior of the HC but changes its value. The EMW leads to a decrease of the $\mathrm{HC}$ at a fixed magnetic field.

In conclusion, the Hall effect in DSSLs subjected to a crossed dc electric field and magnetic field in the presence of an intense EMW has been theoretically studied. In general, the results without the EMW are in accordance with those obtained in some $2 \mathrm{D}$ electron sys-

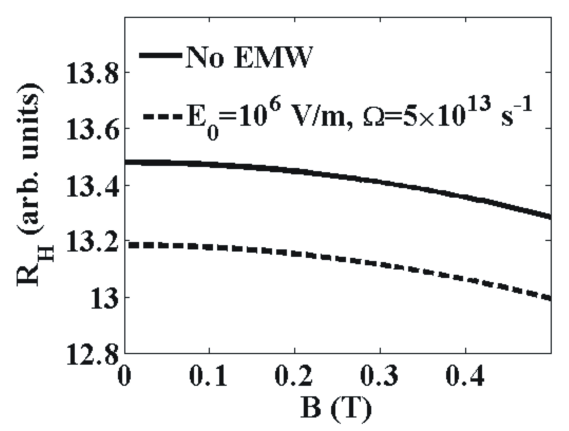

Fig. 4. The HC as function of the magnetic field for two cases: the absence and the presence of the EMW. Here, $n_{D}=$ $7 \times 10^{20} \mathrm{~m}^{-3}$, and $\tau=5.7 \times 10^{-11} \mathrm{~s}$. tems. To our knowledge, the results for the presence of the EMW are new and experimental works are needed to test their validity.

\section{ACKNOWLEDGMENT}

This research is funded by the Vietnam National Foundation for Science and Technology Development (NAFOSTED) under grant number 103.01-2011.18.

\section{REFERENCES}

[1] M. O. Goerbig, "Quantum Hall effect," arXiv:0909.1998v2 [cond-mat.mes-hall], 2009.

[2] N. Q. Bau and B. D. Hoi, J. Korean Phys. Soc. 60 (2012) 59-64.

[3] N. Q. Bau, N. V. Nghia, N. V. Hieu, and B. D. Hoi, Influence of a Strong Electromagnetic Wave (Laser Radiation) on the Hall Coefficient in Doped Semiconductor Superlattices with an In-plane Magnetic Field, PIERS Proceedings, March 25 -28, Taipei, (2013), pp. 416-421.

[4] N. Q. Bau and B. D. Hoi, Int. J. Mod. Phys. B 28 (2014) 1450001 (14 pages).

[5] N. Q. Bau, N. V. Hieu, and N. V. Nhan, Superlattices and Microstruct. 52 (2012) 921-930.

[6] N. Q. Bau and N. V. Hieu, Superlattices and Microstruct. 63 (2013) 121-130.

[7] K. Ploog and G. H. Dohler, Adv. Phys. 32 (1983) 285-359.

[8] X. Chen, J. Phys.: Condens. Matter 9 (1997) 8249-8256.

[9] S. C. Lee, J. Korean Phys. Soc. 51 (2007) 1979-1986.

[10] A. H. Kahn AH and H. P. R. Frederikse, Sol. Stat. Phys. 9 (1959) 257-291.

[11] Vasilopoulos, P., M. Charbonneau, and C. M. Van Vliet, Phys. Rev. B 35 (1987) 1334-1344.

[12] V. É. Kaminskii, Semiconductors 36 (2002) 1276-1282.

[13] F. M. Hashimzade, Kh. A. Hasanov, and M. M. Babayev, Phys. Rev. B 73 (2006) 235349 (8 pp.).

[14] L. Smrčka, P. Vašek, P. Svoboda, N.A. Goncharuk, O. Pacherová, Yu. Krupko, Y. Sheikin, and W. Wegscheider, Physica E 34 (2006) 632-635. 